\title{
Low-Loss Regrowth-Free Long Wavelength Quantum Cascade Lasers
}

\author{
Sinan Gündoğdu ${ }^{\circledR}$, Abdullah Demir ${ }^{\circledR}$, Hadi Sedaghat Pisheh, and Atilla Aydınlı
}

\begin{abstract}
Optical power output is the most sought-after quantity in laser engineering. This is also true for quantum cascade lasers operating especially at long wavelengths. Buried heterostructure cascade lasers with epitaxial regrowth have typically shown the lowest loss due to high current confinement as well as superior lateral thermal conductivity at the expense of complexity and cost. Among the many factors affecting optical output are the widely used passivating materials such as $\mathrm{Si}_{3} \mathrm{~N}_{4}$ and $\mathrm{SiO}_{2}$. These materials have substantial optical absorption in the long wavelength infrared, which results in optical loss reducing the output of the laser. In this letter, we report on quantum cascade lasers with various waveguide widths and cavity lengths using both PECVD grown $\mathrm{Si}_{3} \mathrm{~N}_{4}$ and e-beam evaporated $\mathrm{HfO}_{2}$ as passivating material on the same structure. Their slope efficiency was measured, and the cavity losses for the two lasers were calculated. We show that $\mathrm{HfO}_{2}$ passivated lasers have approximately $5.5 \mathrm{~cm}^{-1}$ lower cavity loss compared to $\mathrm{Si}_{3} \mathrm{~N}_{4}$ passivated lasers. We observe up to $38 \%$ reduction in lasing threshold current, for lasers with $\mathrm{HfO}_{2}$ passivation. We model the losses of the cavity due to both insulator and metal contacts of the lasers using Comsol Multiphysics for various widths. We find that the loss due to absorption in the dielectric is a significant effect for $\mathrm{Si}_{3} \mathrm{~N}_{4}$ passivated lasers and lasers in the 8-12- $\mu \mathrm{m}$ range may benefit from low loss passivation materials such as $\mathrm{HfO}_{2}$. Our results suggest that low-loss long wavelength quantum cascade lasers can be realized without epitaxial overgrowth.
\end{abstract}

Index Terms-Quantum cascade lasers, quantum efficiency, loss, hafnium dioxide, passivation.

\section{INTRODUCTION}

Q UANTUM cascade lasers (QCLs) are unique devices that emit coherent light in a wide spectrum from near-infrared to terahertz frequencies. Their optical output has increased dramatically in the past 20 years from a few $\mathrm{mW}$ to several watts for some wavelength ranges. The requirements of high optical power is ever more present, especially as the lasing wavelength gets longer. In addition to optimization

Manuscript received May 28, 2018; revised September 22, 2018; accepted September 26, 2018. Date of publication October 4, 2018; date of current version November 20, 2018. This work was supported by the Republic of Turkey, Ministry of Science, Industry, and Technology, under Grant 573.STZ.2013-2. (Corresponding author: Sinan Gündoğdu.)

S. Gündoğdu is with the Physics Department, Bilkent University, 06800 Ankara, Turkey (e-mail: gundogdu@fen.bilkent.edu.tr).

A. Demir is with the UNAM-National Nanotechnology Research Center, Institute of Materials Science and Nanotechnology, Bilkent University, 06800 Ankara, Turkey.

H. Sedaghat Pisheh is with the Mechanical Engineering Department, Bilkent University, 06800 Ankara, Turkey.

A. Aydinlı is with the Electrical and Electronics Engineering Department, Uludag University, 16059 Bursa, Turkey.

Color versions of one or more of the figures in this letter are available online at http://ieeexplore.ieee.org.

Digital Object Identifier 10.1109/LPT.2018.2873827 of design and epitaxial growth parameters, the technologies used to fabricate lasers need to be carefully considered. Microfabrication of a standard Fabry-Perot (FP) cavity QCL starts by forming a laser cavity using dry or wet-etching to confine the current for low threshold. To passivate the cavity walls, there are two common approaches [1]; the first one is coating an electrically insulating material such as $\mathrm{SiO}_{2}$ or $\mathrm{Si}_{3} \mathrm{~N}_{4}$ on the ridge walls, typically using PECVD, and the second is regrowth of an insulating epitaxial material, after the formation of the ridge with a semiconductor such as Fe-doped InP, using MOCVD, to fill the trenches that form the cavity walls. Regrown material has the advantage of having low loss and thermal match with the epitaxial material. However, the fabrication of these buried heterostructure (BH) devices is complex and costly. Deposition and processing with $\mathrm{SiO}_{2}$ or $\mathrm{Si}_{3} \mathrm{~N}_{4}$ is a more accessible method, but these materials have relatively high optical absorption coefficients in the 8-12 $\mu \mathrm{m}$ wavelength region [2].

Many factors enter into consideration for the proper choice of a passivation layer. Both the width and intensity of the vibrational mode leading to optical absorption in the passivating layer at the operating wavelength are high on the list. The degree of propagating mode overlap is also significant, as it is critical to the total absorbed power in the passivating layer. Finally, the thermal resistance of the dielectric layer may also be expected to play a role. In previous works, low dissipation DFB devices with power consumption comparable to $\mathrm{BH}$ devices using AlN passivation were demonstrated [3], impact of optical constants and thermal conductivities of $\mathrm{SiO}_{2}, \mathrm{Si}_{3} \mathrm{~N}_{4}$ and $\mathrm{TiO}_{2}$ on the passivation of quantum cascade lasers were theoretically compared, and $\mathrm{SiO}_{2}$ for short wavelengths and $\mathrm{TiO}_{2}$ for long wavelengths into the midwave were recommended [4]. The feasibility of $15 \mu \mathrm{m}$ wide, $3 \mathrm{~mm}$ long double channel pulsed QCLs, insulated by e-beam evaporated $\mathrm{Y}_{2} \mathrm{O}_{3}$ were demonstrated with reports of L-I-V curves, and it was concluded that $\mathrm{SiO}_{2}$ and $\mathrm{Si}_{3} \mathrm{~N}_{4}$ have significant absorption between 8 to $10 \mu \mathrm{m}$ [5]. In another work, they studied the propagation of electromagnetic waves in various dielectrics to calculate absorption loss and optical confinement of QCL waveguides insulated with $\mathrm{SiO}_{2}, \mathrm{Si}_{3} \mathrm{~N}_{4}, \mathrm{As}_{2} \mathrm{~S}_{3}$, and $\mathrm{Ge}_{0.25} \mathrm{Se}_{0.75}$, using the finite element method [6]. In the latter work, $\mathrm{SiO}_{2}$ and $\mathrm{Si}_{3} \mathrm{~N}_{4}$ were compared with the low-loss materials using numerical analysis. However, the loss difference between lasers with different passivation materials was not experimentally measured. $\mathrm{HfO}_{2}$ has not been studied to date as a passivation material for QCLs. 
Various approaches exist in the literature for measurement of loss in QCLs and similar semiconductor lasers. Those include the well-known Hakki-Paoli method [7], a generalized Hakki-Paoli method with Fourier analysis of sub-threshold electroluminescence [8] and analysis of current-power relationship [9], [10]. The first two rely on an analysis of electroluminescence spectra of lasers driven at currents below the lasing threshold. However, in a QCL, transition probabilities between the lasing energy levels depend on the applied voltage, hence, the sub-threshold gain may be significantly different from the gain at the lasing voltage [11]. In the current-power analysis, the relationship between the cavity length and threshold current [10] or slope efficiency [9] is used to deduce the cavity loss. This method is preferred for its relative simplicity and for its ability to yield above-threshold loss. In this study, we compare FP cavity QCLs fabricated with PECVD grown $\mathrm{Si}_{3} \mathrm{~N}_{4}$ and e-beam evaporated $\mathrm{HfO}_{2}$. We measured the slope efficiency and threshold currents of these lasers and from the relationship between the cavity length and slope efficiency, we calculated the cavity losses for the two passivation materials. We also analyzed the optical losses for those materials, including the loss due to contact metals with 2D optical mode analysis using Comsol Multiphysics software.

\section{EXPERIMENTAL}

The laser heterostructure used in this study is similar to three-phonon resonance scheme by Wang et al. [12]. Starting from the top, lattice matched layers are; $100 \mathrm{~nm}$ InGaAs (5E18), $850 \mathrm{~nm} \operatorname{InP}$ (5E18), $2500 \mathrm{~nm} \operatorname{InP}$ (5E16), $200 \mathrm{~nm}$ InGaAs (5E16), $2700 \mathrm{~nm}$ active region, $200 \mathrm{~nm}$ InGaAs (5E16), $2000 \mathrm{~nm} \mathrm{InP} \mathrm{(5E16),} 50 \mathrm{~nm}$ InGaAs (1E18), buffer InP (5E18), substrate InP (5E18). The numbers in the parentheses indicate $\mathrm{Si}$ doping levels. Two pieces of $1.5 \times 1.5 \mathrm{~cm}^{2}$ were cleaved from the same wafer. We formed the waveguides with $12,16,20$ and $24 \mu \mathrm{m}$ width by wet etching on both chips, $6.35 \mu \mathrm{m}$ down to the lower cladding layer. To induce some roughness and improve the adhesion of the passivation layer, top $200 \mathrm{~nm}$ of epitaxial layer was etched with $\mathrm{HBr}: \mathrm{HNO}_{3}: \mathrm{H}_{2} \mathrm{O}$ (1:1:10) solution, everywhere except for the metal contact areas. One sample was coated with approximately $500 \mathrm{~nm}$ $\mathrm{Si}_{3} \mathrm{~N}_{4}$ using PECVD with $180 \mathrm{sccm} 2 \% \mathrm{SiH}_{4}$ in $\mathrm{N}_{2}, 45 \mathrm{sccm}$ $\mathrm{NH}_{3}, 14 \mathrm{~W}$ RF power, 400 mTorr pressure and $250{ }^{\circ} \mathrm{C}$ substrate temperature. The other was coated with approximately $500 \mathrm{~nm} \mathrm{HfO}_{2}$ using e-beam evaporation with a rate of $\approx 0.3 \mathrm{~nm} / \mathrm{sec}$. Metallization contacts were formed by opening windows in the insulators on the top of the waveguides using buffered HF for both chips. Ti/Au (20/200 nm) for the top contacts and $\mathrm{GeAu} / \mathrm{Ni} / \mathrm{Au}(40 / 40 / 150 \mathrm{~nm})$ for the bottom contacts were evaporated using e-beam. Top contacts were electroplated with $5 \mu \mathrm{m}$ gold. Chips were cleaved into $1.8,2.5$ and $3.8 \mathrm{~mm}$ long waveguides and soldered on gold-plated copper mounts with indium paste, epitaxial side up. Lasers were mounted in a liquid nitrogen cooled temperature controlled dewar with $\mathrm{ZnSe}$ window, heatsink temperature was set to $-160{ }^{\circ} \mathrm{C}$. Voltage pulses of $1 \mu \mathrm{s}$ width and $0.1 \%$ duty cycle were applied to the laser. Current through the lasers was measured through a series connected $1 \Omega$ resistor using an oscilloscope. Optical power was measured using a gold integrating sphere with
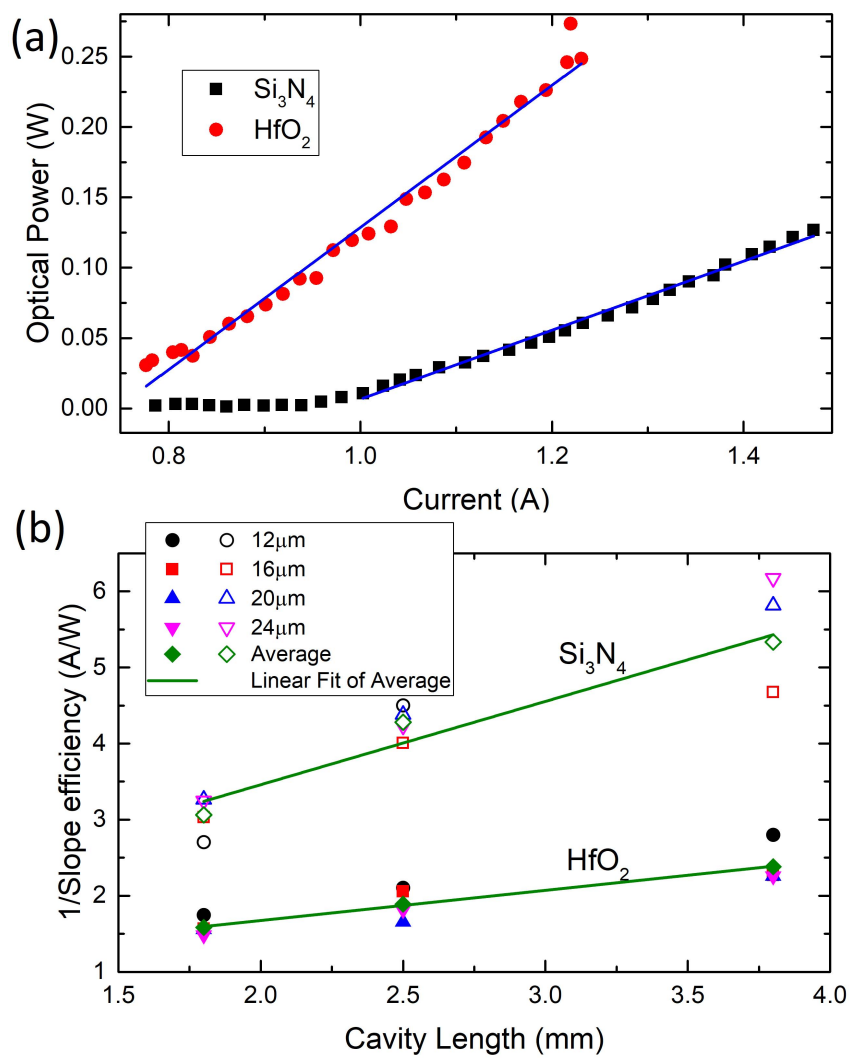

Fig. 1. Optical power as a function of current for $2.5 \mathrm{~mm}$ long $24 \mu \mathrm{m}$ wide lasers passivated with $\mathrm{Si}_{3} \mathrm{~N}_{4}$ and $\mathrm{HfO}_{2}$ (a). Inverse slope efficiency as a function of cavity length (b). $\mathrm{Si}_{3} \mathrm{~N}_{4}$ passivated lasers are shown with hollow symbols, $\mathrm{HfO}_{2}$ passivated ones are shown with solid symbols. Waveguide widths are indicated in the inset. Average inverse slope efficiencies were calculated for each cavity length for both $\mathrm{Si}_{3} \mathrm{~N}_{4}$ and $\mathrm{HfO}_{2}$. Linear fits to the average data are shown with solid lines.

a fast $\mathrm{HgCdTe}$ photodetector connected to an oscilloscope. To measure the absorption coefficients of $\mathrm{Si}_{3} \mathrm{~N}_{4}$ and $\mathrm{HfO}_{2}$, $200 \mathrm{~nm}$ thick films were coated on infrared transparent silicon wafers. The extinction coefficients of the films were deduced from transmission measurement using an FTIR spectrometer.

\section{RESUlTS AND DiscusSiON}

Optical peak power as a function of applied current for $24 \mu \mathrm{m}$ wide and $2.5 \mathrm{~mm}$ long QC lasers is shown in Fig. 1a. Linear fits to the data are shown to guide the eye. Threshold currents were found from the intersection of the fitted lines with the current axis. Slope efficiencies were calculated from the slope of the linear fits. Threshold current for the $\mathrm{Si}_{3} \mathrm{~N}_{4}$ passivated laser is $1.00 \mathrm{~A}$, and it drops to $0.75 \mathrm{~A}$ for the $\mathrm{HfO}_{2}$ passivated one. The slope efficiency of the lasers with $\mathrm{HfO}_{2}$ is larger than that of those passivated with $\mathrm{Si}_{3} \mathrm{~N}_{4}$. We observed that this is the case for other lasers with different widths and lengths as well. Fig. $1 \mathrm{~b}$ shows the inverse slope efficiency as a function of cavity length. The relationship between differential quantum efficiency, $\eta_{d}$, internal quantum efficiency, $\eta_{i}$, optical loss, $\alpha_{i}$, and cavity length, $\mathrm{L}$ is given as [13];

$$
\frac{1}{\eta_{d}}=\frac{\alpha_{i}}{\eta_{i} \ln \left(R_{1} R_{2}\right)^{-1 / 2}} L+\frac{1}{\eta_{i}}
$$



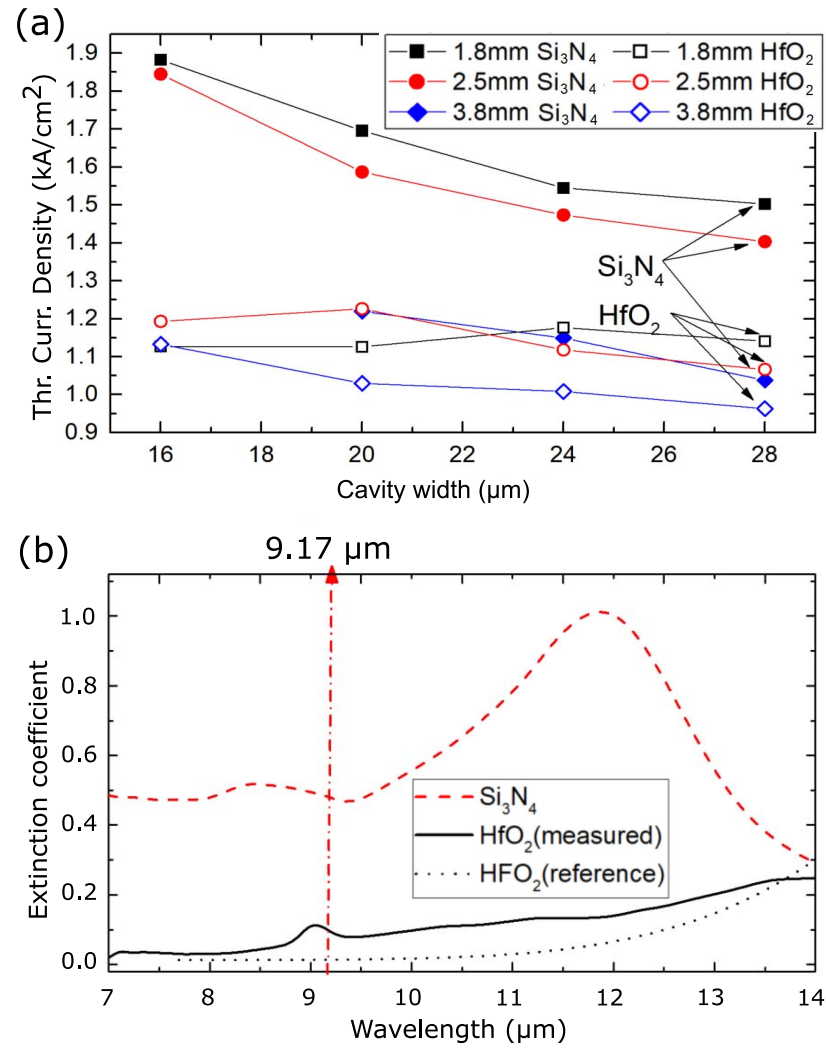

Fig. 2. Threshold current density as a function of cavity width for $\mathrm{Si}_{3} \mathrm{~N}_{4}$ and $\mathrm{HfO}_{2}$ passivation and different cavity lengths (a). Extinction coefficient measured using FTIR absorbance spectra of $\mathrm{Si}_{3} \mathrm{~N}_{4}$ and $\mathrm{HfO}_{2}$ as a function of wavelength (b).

where $R_{1}$ and $R_{2}$ are back and front mirror reflectivities, which, in this case, are equal and $\approx 26 \%$, since the effective indices of the optical modes are $\approx 3.11$ at the operating wavelength. Solid lines are linear fits to the average inverse slope efficiency for different waveguide widths. For $\mathrm{HfO}_{2}$ passivated lasers, optical loss averaged over all ridge widths of $6 \pm 0.5 \mathrm{~cm}^{-1}$ and for $\mathrm{Si}_{3} \mathrm{~N}_{4}, 11.5 \pm 1.0 \mathrm{~cm}^{-1}$ were calculated.

Fig. 2a shows the lasing threshold current densities of the same lasers as Fig. 1, as a function of waveguide width. The threshold current density of the lasers with $\mathrm{Si}_{3} \mathrm{~N}_{4}$ exhibits a strong dependency on waveguide width compared to those with $\mathrm{HfO}_{2}$. This is due to the fact that, in narrower waveguides, optical mode overlap with the passivating dielectric is larger. Therefore, lasers with narrower waveguides are affected more by the optical loss due to the dielectric layer.

To compare the losses due to these two materials, we measured the absorbance spectrum of $\approx 200 \mathrm{~nm}$ thick $\mathrm{HfO}_{2}$ and $\mathrm{Si}_{3} \mathrm{~N}_{4}$ films deposited on infrared transparent silicon. Since the film thickness is much less than the wavelengths of interest, the interference effects can be neglected, and Beer-Lambert law can be used to estimate the extinction coefficient;

$$
1-A=e^{-4 \pi k z / \lambda} \rightarrow k=-\frac{\ln (1-A) \lambda}{4 \pi z}
$$

where $\mathrm{A}$ is the absorbance, $k$ is the extinction coefficient, $z$ is the film thickness and $\lambda$ is the wavelength.
Measured extinction coefficients of the films are shown in Fig. 2b. The extinction coefficient of $\mathrm{Si}_{3} \mathrm{~N}_{4}$ is much larger than that of $\mathrm{HfO}_{2}$ by a factor of 5 at the operating wavelength and peaks at around $11.5 \mu \mathrm{m}$ due to the well-known $\mathrm{Si}-\mathrm{N}$ vibrational modes. The extinction coefficient of $\mathrm{HfO}_{2}$ is less than 0.1 for wavelengths shorter than $10 \mu \mathrm{m}$ and it gets smaller towards shorter wavelengths. The central emission wavelength of our lasers is at $9.17 \mu \mathrm{m}$ and indicated with a red dashed line. At those wavelengths, extinction coefficients are $\mathrm{k}\left(\mathrm{HfO}_{2}\right)=0.095$ and $\mathrm{k}\left(\mathrm{Si}_{3} \mathrm{~N}_{4}\right)=0.48$, and they differ by a factor of 5 . In Fig. $2 b$, the dashed black line is the extinction coefficient of magnetron sputtered $\mathrm{HfO}_{2}$ reported by Bright et al. [14]. This indicates that the extinction coefficient of $\mathrm{HfO}_{2}$ film may even be smaller depending on the coating technique.

To shed light on the role of higher order modes optical loss due to dielectric and metal overcoat numerically, we used Comsol Multiphysics software, for modeling and calculating the complex effective index of the $2 \mathrm{D}$ waveguides for different ridge widths. We introduce the extinction coefficient for the electroplated $\mathrm{Au}$ and the insulator in the form of a complex refractive index. As QCLs lase with TM polarization, calculations were done only for the TM mode. Fig. 3a shows the geometry of the simulation when ridge width is $20 \mu \mathrm{m}$ and Fig. 3b shows the corresponding fundamental mode. We varied the dielectric thickness between $50-1000 \mathrm{~nm}$. From the imaginary part of the effective refractive index, we calculated the loss. Loss, as a function of dielectric thickness for various waveguide widths and dielectric thicknesses is indicated in Fig. 3c. There is a noticeable difference in optical loss between $\mathrm{HfO}_{2}$ and $\mathrm{Si}_{3} \mathrm{~N}_{4}$ passivation. For dielectric thicknesses below $300 \mathrm{~nm}$, loss due to the gold overlayer significantly increases. Above $500 \mathrm{~nm}$, loss converges to a value determined by the extinction coefficient of the insulator.

Averaged over all waveguide widths with $500 \mathrm{~nm}$ dielectric thickness, the simulated fundamental mode loss difference between the two materials is $2.2 \mathrm{~cm}^{-1}$, while the experimental loss difference averaged over all ridge widths is $5.5 \mathrm{~cm}^{-1}$. The difference between simulated and experimental data may be attributed to higher order optical modes neglected in the simulation. The simulated fundamental mode loss also increases as the ridge width decreases, which is consistent with our observation in Fig. 2a. These results are also in agreement with a recent study [5] which indicates that the loss of $\mathrm{Si}_{3} \mathrm{~N}_{4}$ passivated lasers strongly depends on cavity width, compared to low-loss $\mathrm{Y}_{2} \mathrm{O}_{3}$ passivated ones. Fig. 4a displays the first four of the TM mode electric field intensities of a $20 \mu \mathrm{m}$ wide laser. TM0 is the fundamental TM mode and TM1, TM2, TM3 are the higher order modes. Fig. 4c shows the loss of the corresponding modes as a function of waveguide width. Loss with $\mathrm{HfO}_{2}$ passivation is shown with dashed lines and loss with $\mathrm{Si}_{3} \mathrm{~N}_{4}$ passivation is indicated with solid lines. Higher order modes have much higher losses due to larger mode overlap with the dielectric. Therefore, even for wide lasers, loss due to the dielectric is significant as a result of higher order modes. 

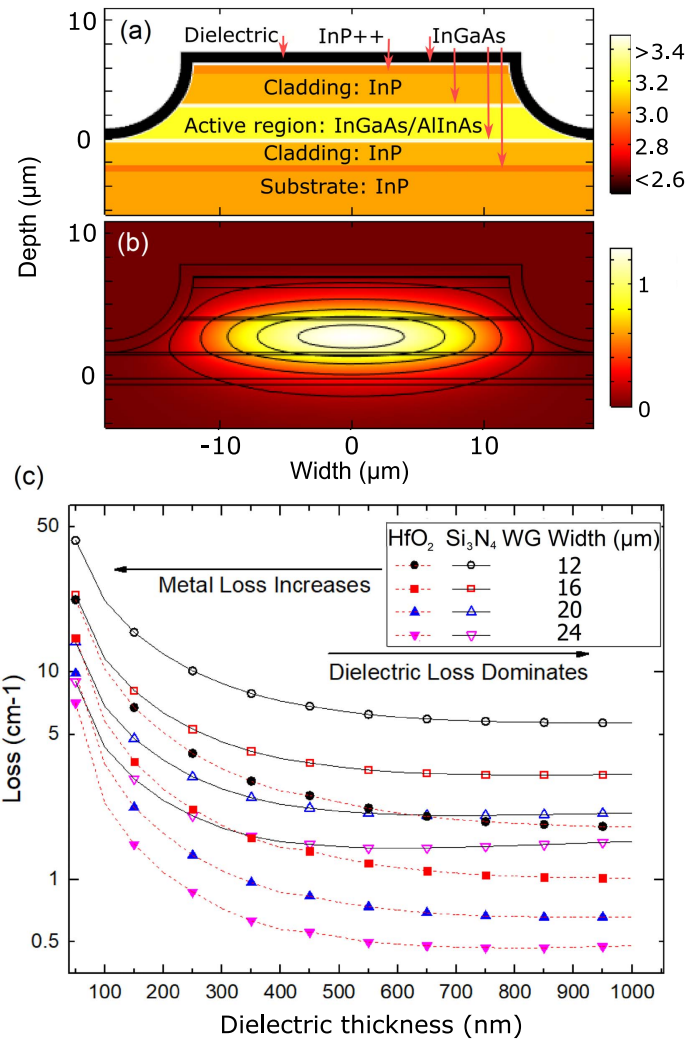

Fig. 3. The structure of the laser we modeled (a), and the calculated fundamental TM mode electric field (b). Calculated losses of the fundamental TM mode of lasers as a function of dielectric thickness (c). Waveguide widths are indicated in the inset. The loss in the case of $\mathrm{Si}_{3} \mathrm{~N}_{4}$ passivation is shown with solid black lines and for $\mathrm{HfO}_{2}$ is shown with dashed red lines. Calculations were done for $9.17 \mu \mathrm{m}$ wavelength. (a)

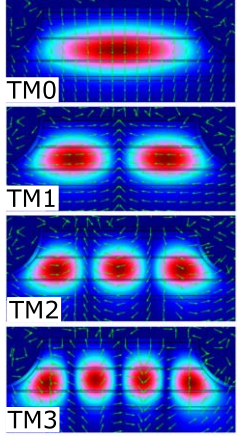

(b)

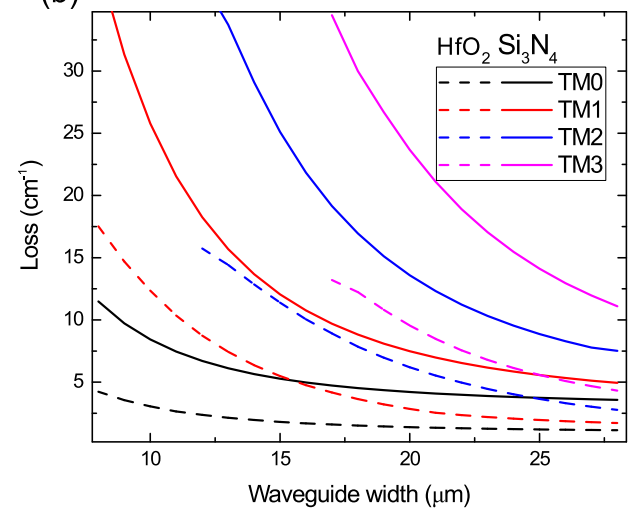

Fig. 4. Electric field intensity and vector map of four TM modes of $20 \mu \mathrm{m}$ wide laser (a), the calculated loss for these modes for $\mathrm{HfO}_{2}$ and $\mathrm{Si}_{3} \mathrm{~N}_{4}$ passivation as a function of waveguide width (b).

Finally, while the bulk thermal conductivity of $\mathrm{HfO}_{2}$ is not as good as that of $\mathrm{Si}_{3} \mathrm{~N}_{4}$, the combined effect of $\mathrm{HfO}_{2}$ and gold layers results in acceptable thermal performance. Indeed, comparison of the numerical solutions of the heat diffusion equation for $\mathrm{HfO}_{2}+\mathrm{Au}, \mathrm{Si}_{3} \mathrm{~N}_{4}+\mathrm{Au}$ and regrown $\mathrm{InP}+\mathrm{Au}$ bilayers, show that for $\mathrm{HFO}_{2}$ passivation, the temperature rise is only $5{ }^{\circ} \mathrm{C}$ higher on the average than the other two.

\section{Conclusion}

We have demonstrated QCLs with passivation by either e-beam evaporated $\mathrm{HfO}_{2}$ or PECVD $\mathrm{Si}_{3} \mathrm{~N}_{4}$ and compared the optical loss. We have shown that experimental loss for $\mathrm{HfO}_{2}$ passivated lasers is almost half that of the $\mathrm{Si}_{3} \mathrm{~N}_{4}$ passivated lasers and lasing threshold current densities are significantly less in the case of $\mathrm{HfO}_{2}$ passivation. Our calculations show that the loss tends to decrease as the waveguides become wider, but even wider lasers have a significant dielectric loss due to higher order modes, Fig. 4. We also find that the thickness of the dielectric layer also plays an important role in the cavity loss and as the dielectric becomes thinner, plasmonic losses caused by the metal contacts increase. To reduce the loss for QCLs that lase in the $8-12 \mu \mathrm{m}$ range, it is advisable to use a low loss dielectric such as $\mathrm{HfO}_{2}$ with approximately $500 \mathrm{~nm}$ thickness. We, therefore, conclude that $\mathrm{HfO}_{2}$ passivation could be a low-loss alternative to conventional passivation materials and a low-cost alternative to regrowth leading to a significant increase in optical power output.

\section{ACKNOWLEDGEMENTS}

The authors thank Prof. Carlo Sirtori for the QCL epitaxial design and valuable discussions and TÜBITAK UME for the loan of integrating sphere for optical power measurements.

\section{REFERENCES}

[1] J. Faist, Quantum Cascade Lasers. London, U.K.: Oxford Univ. Press, 2013, p. 19.

[2] J. Kischkat et al., "Mid-infrared optical properties of thin films of aluminum oxide, titanium dioxide, silicon dioxide, aluminum nitride, and silicon nitride," Appl. Opt., vol. 51, no. 28, pp. 6789-6798, Sep. 2012.

[3] R. M. Briggs et al., "Low-dissipation 7.4- $\mu \mathrm{m}$ single-mode quantum cascade lasers without epitaxial regrowth," Opt. Express, vol. 24, no. 13, pp. 14589-14595, Jun. 2016.

[4] S. Ferré et al., "Comparative study of $\mathrm{SiO}_{2}, \mathrm{Si}_{3} \mathrm{~N}_{4}$ and $\mathrm{TiO}_{2}$ thin films as passivation layers for quantum cascade lasers," Opt. Express, vol. 24, no. 21, pp. 24032-24044, Oct. 2016.

[5] J. Kang et al., "Quantum cascade lasers with $\mathrm{Y}_{2} \mathrm{O}_{3}$ insulation layer operating at $8.1 \mu \mathrm{m}, "$ Opt. Express, vol. 25, no. 16, pp. 19561-19567, Aug. 2017.

[6] G. Rehouma, C. A. Evans, Z. Ikonić, D. Indjin, and P. Harrison, "Comparison of $\mathrm{SiO}_{2}, \mathrm{Si}_{3} \mathrm{~N}_{4}, \mathrm{As}_{2} \mathrm{~S}_{3}$, and $\mathrm{Ge}_{0.25} \mathrm{Se}_{0.75}$ dielectric layers for InP- and GaAs-based material systems for midinfrared quantum cascade laser waveguides," J. Appl. Phys., vol. 106, no. 5, p. 053104, Sep. 2009.

[7] B. W. Hakki and T. L. Paoli, "Gain spectra in GaAs doubleheterostructure injection lasers," J. Appl. Phys., vol. 46, no. 3, pp. 1299-1306, Mar. 1975.

[8] D. Hofstetter and J. Faist, "Measurement of semiconductor laser gain and dispersion curves utilizing Fourier transforms of the emission spectra," IEEE Photon. Technol. Lett., vol. 11, no. 11, pp. 1372-1374, Nov. 1999.

[9] J. Piprek, P. Abraham, and J. E. Bowers, "Cavity length effects on internal loss and quantum efficiency of multiquantum-well lasers," IEEE J. Sel. Topics Quantum Electron., vol. 5, no. 3, pp. 643-647, May 1999.

[10] C. Sirtori et al., "Low-loss Al-free waveguides for unipolar semiconductor lasers," Appl. Phys. Lett., vol. 75, no. 25, pp. 3911-3913, Dec. 1999.

[11] D. G. Revin et al., "Dispersive gain and loss in midinfrared quantum cascade laser," Appl. Phys. Lett., vol. 92, no. 8, p. 081110, Feb. 2008.

[12] Q. J. Wang et al., "High performance quantum cascade lasers based on three-phonon-resonance design," Appl. Phys. Lett., vol. 94, no. 1, p. 011103, Jan. 2009.

[13] L. A. Coldren, S. W. Corzine, and M. L. Mašanović, Diode Lasers and Photonic Integrated Circuits. Hoboken, NJ, USA: Wiley, Mar. 2012, p. 75.

[14] T. J. Bright, J. I. Watjen, Z. M. Zhang, C. Muratore, and A. A. Voevodin, "Optical properties of $\mathrm{HfO}_{2}$ thin films deposited by magnetron sputtering: From the visible to the far-infrared," Thin Solid Films, vol. 520, no. 22, pp. 6793-6802, Sep. 2012. 